\title{
Brain Natriuretic Peptide (BNP) and N-Terminal-proBNP in Cardio-Renal Anemia Syndrome - Difference in Prognostic Ability -
}

\author{
Yoichiro Otaki, MD, PhD; Tetsu Watanabe, MD, PhD; Naohito Sato; \\ Toru Shirata; Shigehiko Kato, MD, PhD; Harutoshi Tamura, MD, PhD; \\ Satoshi Nishiyama, MD, PhD; Takanori Arimoto, MD; Hiroki Takahashi, MD, PhD; \\ Tetsuro Shishido, MD, PhD; Keita Morikane, MD, PhD; Masafumi Watanabe, MD, PhD
}

\begin{abstract}
Background: Cardio-renal anemia syndrome (CRAS) is a growing health problem, with a high mortality rate. Brain natriuretic peptide (BNP) and N-terminal proBNP (NT-proBNP) are well-established diagnostic and prognostic biomarkers of heart failure (HF). The difference in the clinical significance of these biomarkers, however, has not yet been completely elucidated in HF. The aim of the present study was to compare the prognostic ability of BNP and NT-proBNP in HF patients with CRAS.
\end{abstract}

Methods and Results: We measured BNP and NT-proBNP in 492 consecutive HF patients and in 17 control subjects. All patients were prospectively followed up during a median follow-up period of 1,034 days. NT-proBNP/BNP ratio was elevated in HF patients with CRAS compared with those without CRAS and the control subjects. There was no significant difference in the prognostic abilities of BNP and NT-proBNP in all HF patients. The C-index for NT-proBNP for predicting cardiovascular events and mortality, however, was significantly higher than that for BNP in HF patients with CRAS. On multivariate Cox proportional hazards-regression analysis, NT-proBNP, but not BNP, was an independent predictor for clinical outcome in HF with CRAS.

Conclusions: The difference in the prognostic abilities of BNP and NT-proBNP was high in HF patients with CRAS. NT-proBNP had a superior prognostic ability to BNP in HF patients with CRAS.

Key Words: Cardio-renal anemia syndrome; Clinical outcome; Natriuretic peptide

H eart failure (HF) remains a major and increasing public health problem, with a high mortality rate. ${ }^{1}$ Recent reports indicate that cardio-renal anemia syndrome (CRAS) is closely associated with extremely poor outcomes in patients with HF. ${ }^{2-4} \mathrm{HF}$, chronic kidney disease (CKD) and anemia could be exacerbated by each other.

Cardiac biomarkers are generally used for the diagnosis or severity assessment of heart disease. ${ }^{\mathbf{5} 6}$ Natriuretic peptides are synthesized and released from the atrial and ventricular myocardium after the precursor pro-brain natriuretic peptide (BNP) is cleaved into the hormonally active BNP and inactive $\mathrm{N}$-terminal (NT)-proBNP in response to cardiomyocyte stimulation, such as, volume overload, pressure overload, and ischemic injury. ${ }^{7}$ Therefore, BNP and NT-proBNP are produced at equimolar levels at first. Circulating levels of these peptides differ, however, due to different clearance mechanisms, including metabolism and renal excretion. The clearance of BNP depends on neutral endopeptidase-dependent degradation and receptor-mediated degradation. ${ }^{8} \mathrm{BNP}$ acts as an active hormone with vasodilatory and diuretic effects that reduce left ventricular (LV) overload. ${ }^{9}$ In contrast, NT-proBNP is excreted from the kidney without any metabolism and has a longer half-life than BNP. ${ }^{10}$

Despite these differences, both BNP and NT-proBNP are useful biomarkers for the identification of HF patients with a high risk of cardiac events. ${ }^{\mathbf{5 1 1}}$ Natriuretic peptide level is affected by several factors including age, gender, obesity, anemia and kidney function. ${ }^{12-14}$ All of these are major commodities in HF patients. Taking this into consideration, we hypothesized that the clinical significance of natriuretic peptides varies with the existence of commodities. We focused on CRAS, examining its association with natriuretic peptides. We also compared the prognostic abilities of BNP and NT-proBNP in HF patients with CRAS.

Received October 19, 2018; revised manuscript received December 20, 2018; accepted December 23, 2018; J-STAGE Advance Publication released online January 22, 2019 Time for primary review: 33 days

Department of Cardiology, Pulmonology, and Nephrology, Yamagata University School of Medicine, Yamagata (Y.O., T.W., S.K., H. Tamura, S.N., T.A., H. Takahashi, T. Shishido, M.W.); Division of Clinical Laboratory (N.S., T. Shirata), Division of Clinical Laboratory and Infection Control (K.M.), Yamagata University Hospital, Yamagata, Japan

Mailing address: Tetsu Watanabe, MD, PhD, Department of Cardiology, Pulmonology and Nephrology, Yamagata University School of Medicine, 2-2-2 Iida-Nishi, Yamagata 990-9585, Japan. E-mail: tewatana@med.id.yamagata-u.ac.jp

ISSN-2434-0790 All rights are reserved to the Japanese Circulation Society. For permissions, please e-mail: cr@j-circ.or.jp 


\begin{tabular}{|c|c|c|c|c|}
\hline Variables & $\begin{array}{l}\text { All HF patients } \\
(n=492)\end{array}$ & $\begin{array}{c}\text { Non-CRAS } \\
(n=335)\end{array}$ & $\begin{array}{c}\text { CRAS } \\
(n=157)\end{array}$ & P-value \\
\hline Age (years) & $70 \pm 11$ & $68 \pm 12$ & $75 \pm 9$ & $<0.0001$ \\
\hline$M / F$ & $306 / 186$ & $221 / 114$ & $84 / 72$ & 0.0121 \\
\hline $\mathrm{BMI}\left(\mathrm{kg} / \mathrm{m}^{2}\right)$ & $22.2 \pm 3.9$ & $22.4 \pm 4.0$ & $21.7 \pm 3.6$ & 0.1613 \\
\hline Hypertension & $399(81)$ & $267(80)$ & $132(84)$ & 0.2423 \\
\hline Diabetes mellitus & $137(28)$ & $92(27)$ & $45(29)$ & 0.7823 \\
\hline Hyperlipidemia & $135(27)$ & $88(26)$ & $47(30)$ & 0.3954 \\
\hline NYHA II/III/IV & $285 / 141 / 66$ & $208 / 88 / 39$ & $77 / 53 / 27$ & 0.0043 \\
\hline \multicolumn{5}{|l|}{ Etiology } \\
\hline IHD/DCM/Others & $120 / 126 / 246$ & $74 / 93 / 168$ & $46 / 33 / 78$ & 0.1237 \\
\hline \multicolumn{5}{|l|}{ Biochemistry } \\
\hline $\operatorname{LogBNP}(p g / m L)$ & $2.50 \pm 0.54$ & $2.42 \pm 0.56$ & $2.70 \pm 0.49$ & $<0.0001$ \\
\hline LogNT-proBNP (pg/mL) & $3.15 \pm 0.62$ & $2.98 \pm 0.63$ & $3.49 \pm 0.58$ & $<0.0001$ \\
\hline Hemoglobin (mg/dL) & $12.2 \pm 1.9$ & $13.0 \pm 2.0$ & $10.6 \pm 1.5$ & $<0.0001$ \\
\hline eGFR $\left(\mathrm{mL} / \mathrm{min} / 1.73 \mathrm{~m}^{2}\right)$ & $63 \pm 29$ & $74 \pm 22$ & $41 \pm 13$ & $<0.0001$ \\
\hline CKD & $226(46)$ & $69(21)$ & $157(100)$ & $<0.0001$ \\
\hline Anemia & $300(61)$ & $143(43)$ & $157(100)$ & $<0.0001$ \\
\hline \multicolumn{5}{|l|}{ Echocardiography } \\
\hline LVEDD (mm) & $55 \pm 10$ & $55 \pm 10$ & $56 \pm 9$ & 0.4858 \\
\hline LVEF (\%) & $49 \pm 17$ & $49 \pm 17$ & $48 \pm 18$ & 0.6734 \\
\hline \multicolumn{5}{|l|}{ Medication } \\
\hline ACEl and/or ARB & $354(72)$ & $238(71)$ & $116(74)$ & 0.5116 \\
\hline$\beta$-blockers & $322(65)$ & $221(66)$ & $101(64)$ & 0.7216 \\
\hline Loop diuretics & $317(64)$ & $198(59)$ & $119(76)$ & 0.0003 \\
\hline Aldosterone blockers & $144(29)$ & $100(30)$ & $44(28)$ & 0.6766 \\
\hline Statins & $120(24)$ & $73(22)$ & $47(30)$ & 0.0558 \\
\hline
\end{tabular}

Data given as mean $\pm \mathrm{SD}$ or $\mathrm{n}(\%)$. ACEl, angiotensin-converting enzyme inhibitors; $\mathrm{ARB}$, angiotensin II receptor blockers; BMI, body mass index; BNP, brain natriuretic peptide; CKD, chronic kidney disease; CRAS, cardio-renal anemia syndrome; DCM, dilated cardiomyopathy; eGFR, estimated glomerular filtration rate; HF, heart failure; IHD, ischemic heart disease; LVEDD, left ventricular end-diastolic diameter; LVEF, left ventricular ejection fraction; NT-proBNP, N-terminal probrain natriuretic peptide; NYHA, New York Heart Association.

\section{Methods}

\section{Subjects}

This was a prospective observational study, comparing the prognostic abilities of BNP and NT-proBNP between HF patients with and without CRAS. We enrolled 492 patients who were admitted to hospital for the diagnosis or treatment of HF, as well as 17 control subjects without heart disease. The diagnosis of HF was made by 2 cardiologists, who used the generally accepted Framingham criteria, which include a history of dyspnea, symptomatic exercise intolerance with signs of pulmonary congestion or peripheral edema, and radiological or echocardiographic evidence of LV enlargement or dysfunction. ${ }^{15}$

Transthoracic echocardiography was performed by physicians who were blinded to the biochemistry data. The diagnoses of hypertension, diabetes mellitus and hyperlipidemia were established on the basis of medical records or history of medical therapy. Exclusion criteria included acute coronary syndrome (ACS) in the 3 months preceding admission, active hepatic disease, pulmonary disease or malignant disease.

Demographic and clinical data, including age, sex, New York Heart Association (NYHA) functional class and medication at discharge were collected from patient medical records and interviews.

\section{Biochemistry Markers}

Blood samples were obtained in the early morning $\leq 24 \mathrm{~h}$ after admission. These samples were transferred to chilled tubes containing $4.5 \mathrm{mg}$ ethylenediaminetetraacetic acid, disodium salt and aprotinin $(500 \mathrm{U} / \mathrm{mL})$ and were centrifuged at $1,000 \times \mathrm{g}$ for $15 \mathrm{~min}$ at $4^{\circ} \mathrm{C}$. The clarified plasma samples were frozen, stored at $-70^{\circ} \mathrm{C}$, and thawed immediately before the assay was performed. BNP concentration was measured using a commercially available radioimmunoassay specific for human BNP (Shiono RIA BNP assay kit, Shionogi, Tokyo, Japan). ${ }^{16}$

To perform an exact comparison between BNP and NT-proBNP, plasma samples that were obtained simultaneously, were used for NT-proBNP measurement. NTproBNP was measured with an electrochemiluminescence immunoassay: the Elecsys NT-proBNP II on the cobas e602 platform (Roche Diagnostics, Tokyo, Japan).

Estimated glomerular filtration rate (eGFR) was calculated using the Modification of Diet in Renal Disease equation with the Japanese coefficient. ${ }^{17}$

CKD was defined as reduced eGFR $\left(<60 \mathrm{~mL} / \mathrm{min} / 1.73 \mathrm{~m}^{2}\right)$ according to the Kidney Disease Outcomes Quality Initiative (K/DOQI) clinical guidelines. ${ }^{18,19}$ Anemia was defined as hemoglobin $<13 \mathrm{~g} / \mathrm{dL}$ in male subjects and $<12 \mathrm{~g} / \mathrm{dL}$ in female subjects according to the World Health Organization guidelines. CRAS was defined as anemia with CKD. 


\section{Endpoints and Follow-up}

Patients were prospectively followed up for a median of 1,034 days (IQR, 613-1,743 days). Patients were followed up by telephone or medical record twice a year until 2,555 days after discharge. Nine patients were lost to follow-up due to them moving or being unable to be contacted. The primary endpoints were cardiac events: progressive HF requiring rehospitalization; ACS; and cardiac death defined as death due to progressive HF, ACS or sudden cardiac death. Sudden cardiac death was defined as death without definite warning symptoms or signs. Sudden cardiac death was diagnosed by the attending physician after patients were transported to the emergency department. The secondary endpoint was cardiovascular death. There were 16 noncardiac deaths during the follow-up period.

The study was approved by the institutional ethics committee and all patients provided written informed consent to participate.

\section{Statistical Analysis}

Continuous variable normality was checked using ShapiroWilk test. Due to the non-normal distribution of BNP and NT-proBNP, we used $\log _{10}$-transformed BNP and NT-proBNP in all analyses. All data are expressed as mean \pm SD. The NT-proBNP/BNP ratio was calculated and used for assessing the differences in the effects of BNP and NT-proBNP. ${ }^{20}$ The t-test and chi-squared test were used to compare continuous and categorical variables, respectively. Multivariate logistic analysis was performed to identify the factors associated with CRAS. Receiver operating characteristics (ROC) curve analysis for cardiac events and mortality was performed to compare the prognostic abilities of BNP, NT-proBNP and NT-proBNP/ $\mathrm{BNP}$ ratio. Cox proportional hazards analysis was carried out to identify independent predictors for cardiac events. Predictors that were significant on univariate analysis were used for multivariate analysis. Cardiac event-free curves were constructed according to the Kaplan-Meier method and compared using log-rank test. $\mathrm{P}<0.05$ was considered significant. Statistical analysis was performed using JMP version 12 (SAS Institute, Cary, NC, USA) and R 3.0.2 with additional packages including Rcmdr, Epi, and $\mathrm{pROC}$.

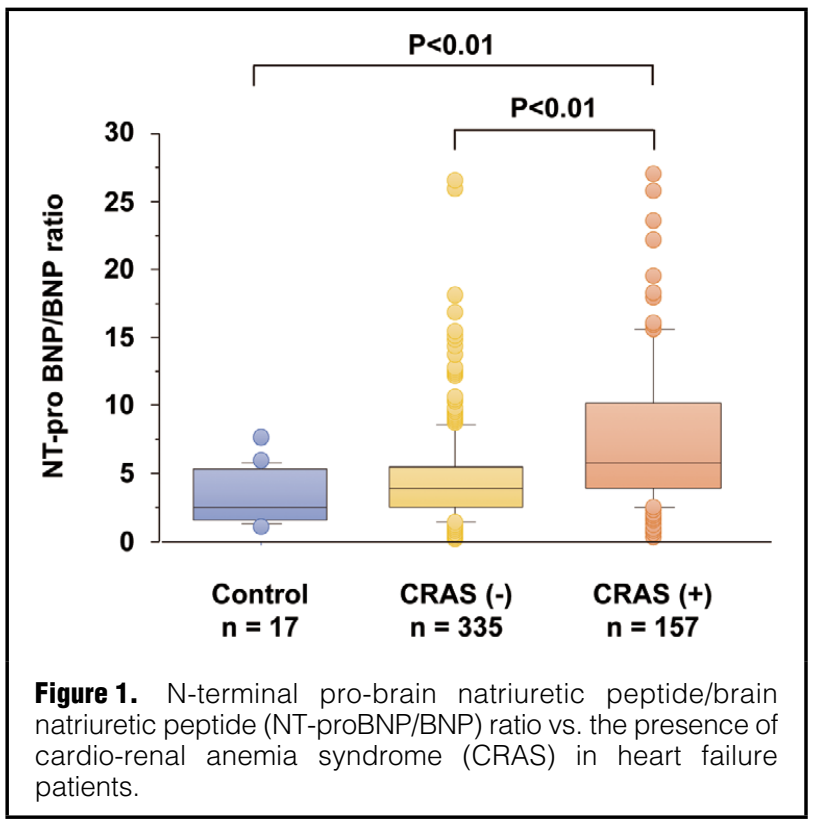

\section{Results}

\section{HF Subject Characteristics and Presence of CRAS}

The baseline clinical characteristics of all HF patients and comparisons of clinical characteristics between those with and without CRAS are given in Table 1. Hypertension, diabetes mellitus, and hyperlipidemia were identified in $399(81 \%), 137(28 \%)$, and $135(27 \%)$, respectively. The etiologies of HF were ischemic heart disease in 120 patients, dilated cardiomyopathy in 126 , and others in the remaining 246 patients. Mean log-BNP and log-NTproBNP were 2.50 and 3.15 , respectively. CKD, anemia, and CRAS were identified in $226(46 \%), 300(61 \%)$, and $157(31 \%)$, respectively.

Patients with CRAS were older, more patients were female, and had a more severe NYHA functional class than those without it. Patients with CRAS had higher $\log$-BNP and log-NT-proBNP and had lower hemoglobin and eGFR than those without it. There were no significant differences in body mass index, prevalence of hypertension, diabetes mellitus, or hyperlipidemia, echocardiographic

\begin{tabular}{|c|c|c|c|c|c|c|}
\hline \multirow{2}{*}{ Variables } & \multicolumn{3}{|c|}{ Univariate analysis } & \multicolumn{3}{|c|}{ Multivariate analysis } \\
\hline & OR & $95 \% \mathrm{Cl}$ & P-value & OR & $95 \% \mathrm{Cl}$ & P-value \\
\hline Age & 1.065 & $1.044-1.088$ & $<0.0001$ & 1.055 & $1.031-1.080$ & $<0.0001$ \\
\hline Female vs. Male & 1.642 & $1.115-2.418$ & 0.0121 & 1.602 & $1.007-2.551$ & 0.0464 \\
\hline Hypertension & 1.345 & $0.822-2.259$ & 0.2423 & 1.023 & $0.548-1.870$ & 0.9407 \\
\hline Diabetes mellitus & 1.061 & $0.639-1.611$ & 0.7823 & 1.053 & $0.622-1.764$ & 0.8470 \\
\hline Hyperlipidemia & 1.199 & $0.785-1.819$ & 0.3976 & 1.336 & $0.805-2.209$ & 0.2607 \\
\hline NYHA III/IV vs. II & 1.702 & $1.161-2.499$ & 0.0065 & 1.354 & $0.858-2.137$ & 0.1915 \\
\hline NT-proBNP/BNP ratio & 1.135 & $1.085-1.193$ & $<0.0001$ & 1.111 & $1.061-1.169$ & $<0.0001$ \\
\hline LVEDD ${ }^{\dagger}$ & 1.075 & $0.877-1.320$ & 0.4852 & 1.293 & $0.972-1.730$ & 0.0783 \\
\hline LVEF' $^{\dagger}$ & 0.959 & $0.788-1.167$ & 0.6725 & 0.858 & $0.649-1.132$ & 0.2793 \\
\hline Loop diuretics use & 2.166 & $1.427-3.345$ & 0.0002 & 2.045 & $1.234-3.455$ & 0.0052 \\
\hline
\end{tabular}

tPer 1-SD increase. $\mathrm{Cl}$, confidence interval; OR, odds ratio. Other abbreviations as in Table 1. 

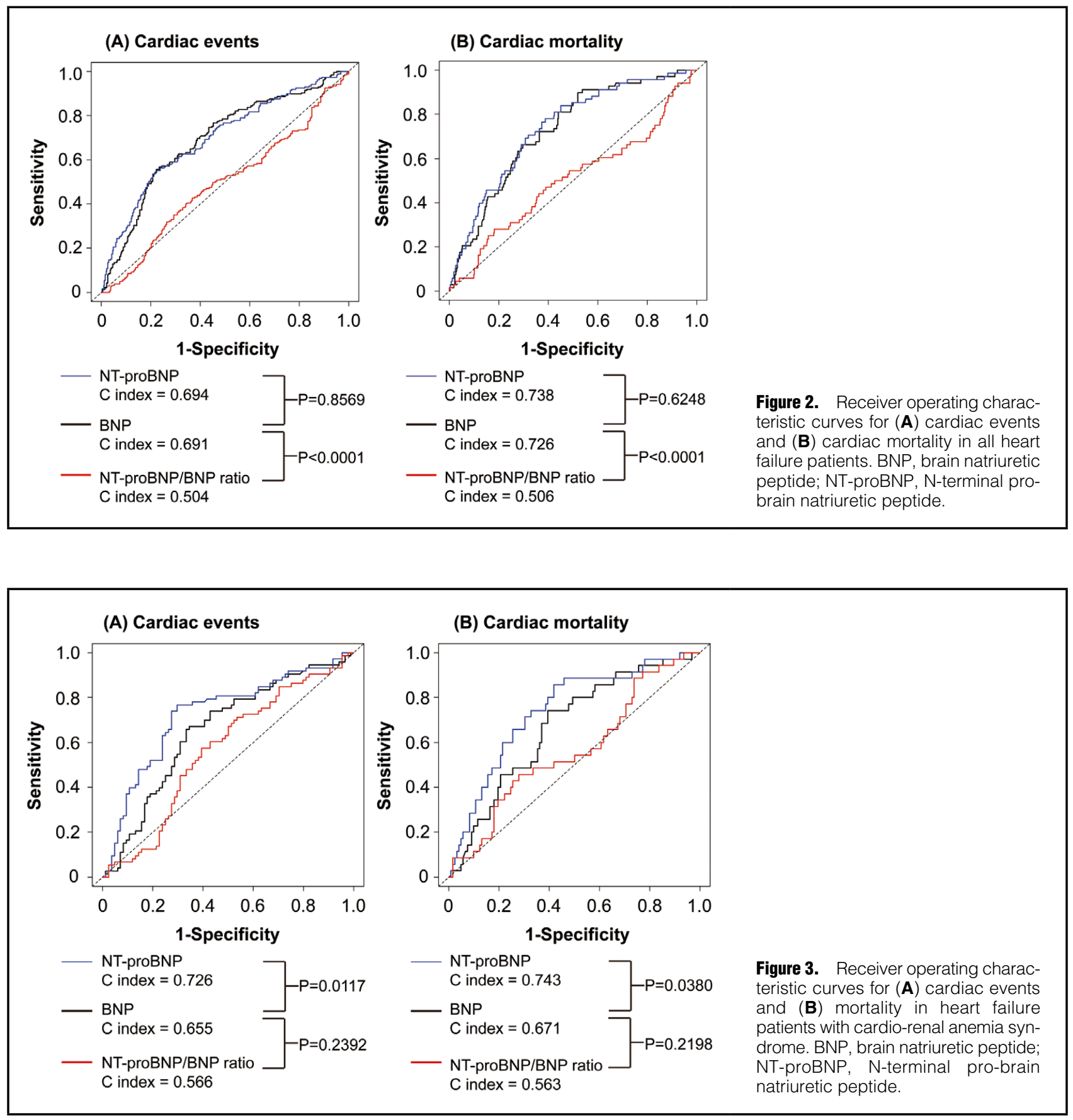

parameters, or medication excluding loop diuretics, between patients with and without CRAS.

\section{NT-proBNP/BNP Ratio and CRAS}

To determine the impact of CRAS on natriuretic peptide level, we calculated the NT-proBNP/BNP ratio. As shown in Figure 1, NT-proBNP/BNP ratio was higher in patients with CRAS than in those without CRAS, as well as in the control subjects.

Next, to determine whether NT-proBNP/BNP ratio was related to CRAS, we performed univariate and multivariate logistic analyses for the presence of CRAS in HF patients. As shown in Table 2, NT-proBNP/BNP ratio was significantly associated with CRAS. Female sex, NYHA func- tional class and loop diuretics use were also related to CRAS. On multivariate logistic analysis, a high NTproBNP/BNP ratio was independently related to the presence of CRAS, suggesting that the difference in circulating BNP and NT-proBNP was greater in CRAS patients than in those without it.

\section{Clinical Outcome and Natriuretic Peptides}

There were 185 cardiac events and 68 cardiac deaths during the follow-up period. The prognostic ability of BNP, NTproBNP and NT-proBNP/BNP ratio was compared in all HF patients using ROC curves for cardiac events and mortality (Supplementary Figure 1). As shown in Figure 2, there were no significant differences in $\mathrm{C}$ index between 
(A)

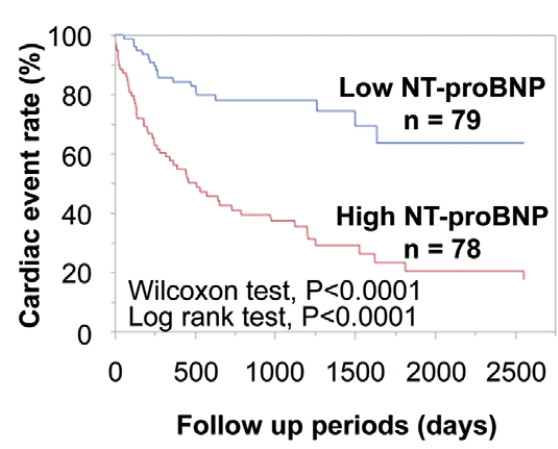

No. at risk

Low NT-proBNP

$\begin{array}{lllll}79 & 56 & 32 & 16 & 9\end{array}$

High NT-proBNP

$\begin{array}{lllll}78 & 34 & 20 & 12 & 8\end{array}$
(B)

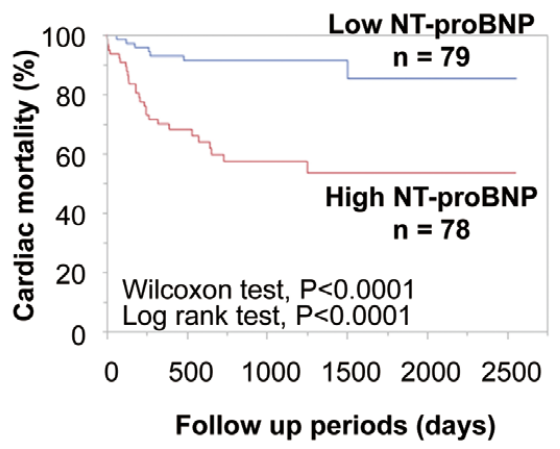

No. at risk

Low NT-proBNP

$\begin{array}{lllll}79 & 56 & 32 & 16 & 9\end{array}$

High NT-proBNP

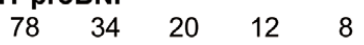

Figure 4. Kaplan-Meier curves for $(\mathbf{A})$ cardiac event rate and (B) cardiac mortality in heart failure patients with cardio-renal anemia syndrome according to N-terminal pro-brain natriuretic peptide (NT-proBNP) level.

\begin{tabular}{|c|c|c|c|c|c|c|}
\hline \multirow{2}{*}{ Variables } & \multicolumn{3}{|c|}{ Univariate analysis } & \multicolumn{3}{|c|}{ Multivariate analysis } \\
\hline & HR & $95 \% \mathrm{Cl}$ & P-value & HR & $95 \% \mathrm{Cl}$ & P-value \\
\hline Age & 0.997 & $0.975-1.027$ & 0.9835 & 1.016 & $0.989-1.044$ & 0.2385 \\
\hline Female vs. Male & 0.429 & $0.257-0.696$ & 0.0005 & 0.457 & $0.265-0.768$ & 0.0029 \\
\hline Hypertension & 1.142 & $0.614-2.369$ & 0.6914 & & & \\
\hline Diabetes mellitus & 1.190 & $0.715-1.933$ & 0.4870 & & & \\
\hline Hyperlipidemia & 1.043 & $0.619-1.699$ & 0.8697 & & & \\
\hline NYHA III/IV vs. II & 1.806 & $1.135-2.917$ & 0.0124 & 1.404 & $0.849-2.351$ & 0.1866 \\
\hline $\mathrm{eGFR}^{\dagger}$ & 0.531 & $0.347-0.832$ & 0.0045 & 0.825 & $0.449-1.527$ & 0.5376 \\
\hline Hemoglobin ${ }^{\dagger}$ & 0.910 & $0.692-1.214$ & 0.5125 & 0.788 & $0.567-1.646$ & 0.1470 \\
\hline Log BNP' & 1.721 & $1.403-2.135$ & $<0.0001$ & 1.151 & $0.709-1.898$ & 0.5763 \\
\hline Log NT-proBNP' ${ }^{\dagger}$ & 2.447 & $1.946-3.136$ & $<0.0001$ & 1.820 & $1.125-2.973$ & 0.0156 \\
\hline NT-proBNP/BNP ratio & 0.759 & $0.309-1.818$ & 0.5426 & & & \\
\hline LVEDD $^{\dagger}$ & 1.200 & $0.919-1.572$ & 0.1796 & & & \\
\hline LVEF$^{\dagger}$ & 0.618 & $0.234-1.609$ & 0.3275 & & & \\
\hline Loop diuretics use & 1.580 & $0.905-2.958$ & 0.1270 & 1.936 & $1.049-3.873$ & 0.0460 \\
\hline
\end{tabular}

tPer 1-SD increase. HR, hazard ratio. Other abbreviations as in Tables 1,2.

BNP and NT-proBNP. The C indices for NT-proBNP/BNP ratio for cardiac events and mortality were significantly lower than those for BNP and NT-proBNP in all HF patients. In addition, we compared the prognostic ability of BNP and NT-proBNP in HF patients with CKD, those without CKD, those with anemia, and those without anemia (Supplementary Figure 2). Interestingly, the $\mathrm{C}$ index for NT-proBNP for cardiac events, but not cardiac mortality, was significantly greater than that for BNP in HF patients with CKD. In contrast, the $\mathrm{C}$ index for NTproBNP for cardiac mortality, but not cardiac events, was significantly greater than that for BNP in HF patients with anemia.

Next, we compared the prognostic ability of BNP, NT-proBNP and NT-proBNP/BNP ratio in HF patients with CRAS on ROC curves for cardiac events and mortality. The C indices for NT-proBNP for cardiac events and mortality were significantly greater than those for BNP, suggesting that NT-proBNP was a superior prognostic marker to BNP in HF patients with CRAS (Figure 3). The $\mathrm{C}$ indices for NT-proBNP/BNP ratio for cardiac events and mortality were significantly lower than those for BNP and NT-proBNP in HF patients with CRAS. Next, HF patients with CRAS were divided into 2 groups, based on median NT-proBNP. On Kaplan-Meier analysis, patients with high NT-proBNP had higher rates of cardiac events and mortality than those with low NT-proBNP, in HF patients with CRAS (Figure 4).

To examine whether NT-proBNP could predict clinical outcomes in patients with CRAS, we performed univariate 
and multivariate Cox proportional-hazards regression analyses. As shown in Table 3, on univariate Cox proportional-hazards regression analysis both BNP and NTproBNP, but not NT-proBNP/BNP ratio, were significantly associated with cardiac events. Also, NYHA functional class and eGFR were related to cardiac events in CRAS patients. On multivariate Cox proportional-hazards regression analysis, NT-proBNP, but not BNP, was an independent predictor for cardiac events after adjustment for age, sex, NYHA functional class, eGFR, hemoglobin, $\mathrm{BNP}$ and loop diuretic use.

\section{Discussion}

The novel findings from the present study were: (1) patients with CRAS had a higher NT-proBNP/BNP ratio than those without it; (2) on multivariate logistic analysis a high NT-proBNP/BNP ratio was associated with the presence of CRAS; (3) although $\mathrm{C}$ index was almost identical in all HF patients, the $\mathrm{C}$ index of NT-proBNP was significantly greater than that of BNP in HF patients with CRAS; and (4) NT-proBNP, but not BNP, was an independent predictor for cardiac events and mortality in $\mathrm{HF}$ patients with CRAS

The clinical application of natriuretic peptides was expanded, from a diagnostic marker for HF, to a prognostic marker for cardiovascular disease in a broad spectrum of heart disease. It is worth examining whether natriuretic peptides serve as a prognostic marker for clinical outcomes in patients with severe heart disease, that is, CRAS.

Anemia and kidney dysfunction are common comorbidities in HF patients, the prevalence of which reaches $30-50 \%$. In the present study, CRAS was identified in $31 \%$ of HF patients and was associated with poor clinical outcome, similar to past reports (Supplementary Figure 3).21 Intriguingly, anemia and kidney dysfunction are independently associated with natriuretic peptide elevation in $\mathrm{HF}$ patients. ${ }^{22}$ A recent study indicated that the consideration of anemia is recommended for physicians when assessing natriuretic peptide levels in patients with suspected HF.23 One of the possible mechanisms by which anemia increases natriuretic peptide level is that anemia leads to an elevated plasma volume, resulting in LV remodeling. ${ }^{24}$ Therefore, anemia increases natriuretic peptide levels through augmented production.

In contrast, kidney dysfunction increases natriuretic peptide level through impaired excretion. The cut-off points of natriuretic peptide level for the diagnosis of $\mathrm{HF}$ rise in line with CKD level, and an increase in NT-proBNP was relatively higher compared with that in $\mathrm{BNP}$, with an advancing CKD stage in patients with dyspnea, despite baseline production by the ventricular myocytes being at equimolar levels. ${ }^{25}$ In addition, Takase and Dohi reported that deterioration in kidney function has a greater impact on NT-proBNP than on BNP. They also reported, however, that NT-proBNP/BNP ratio is preserved in moderate kidney dysfunction due to preserved NT-proBNP excretion. ${ }^{\mathbf{2 6}}$ Given that patients with CRAS had moderate kidney dysfunction (eGFR, $41 \mathrm{~mL} / \mathrm{min} / 1.73 \mathrm{~m}^{2}$ ) in the present study, other mechanisms as well as kidney dysfunction may be involved in the elevation of NT-proBNP/BNP ratio. Therefore, the increased difference in circulating BNP and NT-proBNP may result from augmentation of their production, impaired NT-proBNP excretion and BNP metabolism, in patients with CRAS.
Recently, LCZ696, a new therapeutic drug for HF, has been given a class I recommendation for HF patients with reduced ejection fraction (EF), based on the American Heart Association and European Society of Cardiology guidelines. ${ }^{27}$ The Prospective Comparison of angiotensinreceptor-neprilysin inhibitor (ARNI) with angiotensinconverting enzyme inhibitor (ACEI) to Determine Impact on Global Mortality and Morbidity in Heart Failure (PARADIGM-HF) trial demonstrated that BNP increased and NT-proBNP decreased after use of LCZ696. ${ }^{28}$ Given that BNP is a substrate for LCZ696, the diagnostic and prognostic roles of NT-proBNP are gaining attention in the field of HF patient treatment.

Although several reports have compared the prognostic ability of BNP and NT-proBNP, the results were controversial. The Valsartan Heart Failure (Val-HeFT) Data, which is the largest study discussing this issue, showed that NT-proBNP was superior to BNP in predicting cardiac events in HF patients. ${ }^{11}$ In the present study, however, their prognostic abilities were equal in all HF patients, similar to other previous studies. ${ }^{\mathbf{2 9}, 30}$ These differences may be explained by the patient clinical background and the sample size. Importantly, we showed that NT-proBNP, but not BNP, was independently associated with cardiac events, and that $\mathrm{C}$ indices for NT-proBNP for cardiac events and mortality were significantly higher than those for BNP in HF patients with CRAS, despite the small sample size. Notably, NT-proBNP is associated with clinical outcome and had superior prognostic value in patients with hemodialysis, ${ }^{31,32}$ who generally had comorbid anemia and kidney dysfunction. Therefore, CRAS is an important comorbidity that affects natriuretic peptide level, and, potentially, prognostic ability.

The clinical perspective of the present study is that NT-proBNP measurement is useful and could be a feasible marker for cardiac events in HF patients with CRAS, having superiority to BNP. Physicians should be aware of variables that affect natriuretic peptide level and appropriately select natriuretic peptides when assessing severity and clinical outcomes in HF patients.

\section{Study Limitations}

First, BNP increases during carperitide infusion, but NT-proBNP does not vary with it, ${ }^{33}$ suggesting that NTproBNP/BNP ratio is modulated by carperitide infusion during acute-phase HF. Given that carperitide is usually used in CRAS, we may have underestimated the NTproBNP/BNP ratio, despite there being a significant difference. Second, because this was a single-center study, the number of patients was relatively small. Third, we did not assess the cause of anemia. Thus, we did not distinguish between iron deficiency anemia, kidney anemia, and other causes. Fourth, natriuretic peptides at discharge were also associated with cardiac events in patients with $\mathrm{CHF}$. Further studies are needed to determine whether NTproBNP at discharge also has superior prognostic ability to BNP at discharge in HF patients with CRAS. Finally, although the mean LVEF in the present study was equivalent to that reported in the Japanese HF study and HF registry, ${ }^{34}$ it was relatively high compared with that seen in Western countries. ${ }^{35}$

\section{Conclusions}

Natriuretic peptide level was affected by CRAS; the differ- 
ence in BNP and NT-proBNP was high in HF patients with CRAS. This difference may result in the effectiveness of NT-proBNP in predicting clinical outcomes in HF patients with CRAS compared with BNP. NT-proBNP, but not BNP, could provide useful clinical information in HF patients with CRAS. Physicians should pay attention to CRAS and correctly select suitable natriuretic peptides for the identification of high-risk patients.

\section{Disclosures}

The authors declare no conflicts of interest.

\section{References}

1. Bui AL, Horwich TB, Fonarow GC. Epidemiology and risk profile of heart failure. Nat Rev Cardiol 2011; 8: 30-41.

2. Goh CY, Vizzi G, De Cal M, Ronco C. Cardiorenal syndrome: A complex series of combined heart/kidney disorders. Contrib Nephrol 2011; 174: $33-45$.

3. Ronco C, Haapio M, House AA, Anavekar N, Bellomo R Cardiorenal syndrome. J Am Coll Cardiol 2008; 52: 1527-1539.

4. Otaki Y, Watanabe T, Takahashi H, Narumi T, Kadowaki S, Honda $\mathrm{Y}$, et al. Association of renal tubular damage with cardiorenal anemia syndrome in patients with heart failure. Int $J$ Cardiol 2014; 173: $222-228$.

5. Tsutamoto T, Wada A, Maeda K, Hisanaga T, Maeda Y, Fukai $\mathrm{D}$, et al. Attenuation of compensation of endogenous cardiac natriuretic peptide system in chronic heart failure: Prognostic role of plasma brain natriuretic peptide concentration in patients with chronic symptomatic left ventricular dysfunction. Circulation 1997; 96: 509-516

6. Ahmad T, Fiuzat M, Felker GM, O'Connor C. Novel biomarkers in chronic heart failure. Nat Rev Cardiol 2012; 9: 347-359.

7. Nishikimi T, Nakagawa $Y$, Minamino N, Ikeda M, Tabei K, Fujishima A, et al. Pro-B-type natriuretic peptide is cleaved intracellularly: Impact of distance between O-glycosylation and cleavage sites. Am J Physiol Regul Integr Comp Physiol 2015; 309: R639-R649.

8. Potter LR. Natriuretic peptide metabolism, clearance and degradation. FEBS J 2011; 278: 1808-1817.

9. de Lemos JA, McGuire DK, Drazner MH. B-type natriuretic peptide in cardiovascular disease. Lancet 2003; 362: 316-322.

10. Levin ER, Gardner DG, Samson WK. Natriuretic peptides. $N$ Engl J Med 1998; 339: 321-328.

11. Masson S, Latini R, Anand IS, Vago T, Angelici L, Barlera S, et al. Direct comparison of B-type natriuretic peptide (BNP) and amino-terminal proBNP in a large population of patients with chronic and symptomatic heart failure: The Valsartan Heart Failure (Val-HeFT) data. Clin Chem 2006; 52: 1528-1538.

12. Redfield MM, Rodeheffer RJ, Jacobsen SJ, Mahoney DW, Bailey KR, Burnett JC Jr. Plasma brain natriuretic peptide concentration: Impact of age and gender. J Am Coll Cardiol 2002; 40: 976-982.

13. Tsutamoto T, Sakai H, Ishikawa C, Fujii M, Tanaka T, Yamamoto T, et al. Direct comparison of transcardiac difference between brain natriuretic peptide (BNP) and N-terminal pro-BNP in patients with chronic heart failure. Eur J Heart Fail 2007; 9: $667-673$

14. Tsuji H, Nishino N, Kimura Y, Yamada K, Nukui M, Yamamoto $\mathrm{S}$, et al. Haemoglobin level influences plasma brain natriuretic peptide concentration. Acta Cardiol 2004; 59: 527-531.

15. McKee PA, Castelli WP, McNamara PM, Kannel WB. The natural history of congestive heart failure: The Framingham study. N Engl J Med 1971; 285: 1441-1446.

16. Otaki Y, Watanabe T, Takahashi H, Hirayama A, Narumi T, Kadowaki S, et al. Association of heart-type fatty acid-binding protein with cardiovascular risk factors and all-cause mortality in the general population: The Takahata Study. PLoS One 2014; 9: e94834.

17. Matsuo S, Imai E, Horio M, Yasuda Y, Tomita K, Nitta K, et al. Revised equations for estimated GFR from serum creatinine in Japan. Am J Kidney Dis 2009; 53: 982-992.

18. Eckardt KU, Berns JS, Rocco MV, Kasiske BL. Definition and classification of CKD: The debate should be about patient prognosis: A position statement from KDOQI and KDIGO. Am J Kidney Dis 2009; 53: 915-920.

19. National Kidney Foundation. K/DOQI clinical practice guidelines for chronic kidney disease: Evaluation, classification, and strati- fication. Am J Kidney Dis 2002; 39: S1-S266.

20. Tsutamoto T, Sakai H, Nishiyama K, Tanaka T, Fujii M, Yamamoto T, et al. Direct comparison of transcardiac increase in brain natriuretic peptide (BNP) and N-terminal proBNP and prognosis in patients with chronic heart failure. Circ $J$ 2007; 71: $1873-1878$.

21. Lu KJ, Kearney LG, Hare DL, Ord M, Toia D, Jones E, et al. Cardiorenal anemia syndrome as a prognosticator for death in heart failure. Am J Cardiol 2013; 111: 1187-1191.

22. Hogenhuis J, Voors AA, Jaarsma T, Hoes AW, Hillege HL, Kragten JA, et al. Anaemia and renal dysfunction are independently associated with BNP and NT-proBNP levels in patients with heart failure. Eur J Heart Fail 2007; 9: 787-794.

23. Karakoyun I, Colak A, Arslan FD, Hasturk AG, Duman C. Anemia considerations when assessing natriuretic peptide levels in ED patients. Am J Emerg Med 2017; 35: 1677-1681.

24. Anand IS, Chandrashekhar Y, Ferrari R, Poole-Wilson PA, Harris PC. Pathogenesis of oedema in chronic severe anaemia: Studies of body water and sodium, renal function, haemodynamic variables, and plasma hormones. Br Heart J 1993; 70: 357-362.

25. Chenevier-Gobeaux C, Claessens YE, Voyer S, Desmoulins D, Ekindjian OG. Influence of renal function on N-terminal probrain natriuretic peptide (NT-proBNP) in patients admitted for dyspnoea in the Emergency Department: Comparison with brain natriuretic peptide (BNP). Clin Chim Acta 2005; 361: 167-175.

26. Takase H, Dohi Y. Kidney function crucially affects B-type natriuretic peptide (BNP), N-terminal proBNP and their relationship. Eur J Clin Invest 2014; 44: 303-308.

27. Ponikowski P, Voors AA, Anker SD, Bueno H, Cleland JG, Coats AJ, et al. 2016 ESC Guidelines for the diagnosis and treatment of acute and chronic heart failure: The Task Force for the diagnosis and treatment of acute and chronic heart failure of the European Society of Cardiology (ESC) Developed with the special contribution of the Heart Failure Association (HFA) of the ESC. Eur Heart J 2016; 37: 2129-2200.

28. Packer M, McMurray JJ, Desai AS, Gong J, Lefkowitz MP, Rizkala AR, et al. Angiotensin receptor neprilysin inhibition compared with enalapril on the risk of clinical progression in surviving patients with heart failure. Circulation 2015; 131: $54-61$.

29. Alehagen U, Lindstedt G, Levin LA, Dahlstrom U. Risk of cardiovascular death in elderly patients with possible heart failure. B-type natriuretic peptide (BNP) and the aminoterminal fragment of ProBNP (N-terminal proBNP) as prognostic indicators in a 6-year follow-up of a primary care population. Int $J$ Cardiol 2005; 100: 125-133.

30. Hulsmann M, Berger R, Mortl D, Gore O, Meyer B, Pacher R Incidence of normal values of natriuretic peptides in patients with chronic heart failure and impact on survival: A direct comparison of N-terminal atrial natriuretic peptide, N-terminal brain natriuretic peptide and brain natriuretic peptide. Eur $J$ Heart Fail 2005; 7: 552-556

31. Winkler K, Wanner C, Drechsler C, Lilienthal J, Marz W, Krane $\mathrm{V}$. Change in N-terminal-pro-B-type-natriuretic-peptide and the risk of sudden death, stroke, myocardial infarction, and all-cause mortality in diabetic dialysis patients. Eur Heart J 2008; 29: 2092-2099.

32. Sun L, Sun Y, Zhao X, Xu C, Chen D, Li L, et al. Predictive role of BNP and NT-proBNP in hemodialysis patients. Nephron Clin Pract 2008; 110: c178-c184.

33. Nishiyama K, Tsutamoto T, Tanaka T, Fujii M, Yamamoto T, Yamaji M, et al. Plasma NT-proBNP as a more reliable biomarker of endogenous cardiac natriuretic peptides than BNP during carperitide infusion. Int Heart J 2009; 50: 183-190.

34. Shiba N, Nochioka K, Miura M, Kohno H, Shimokawa H. Trend of westernization of etiology and clinical characteristics of heart failure patients in Japan: First report from the CHART-2 study. Circ J 2011; 75: 823-833.

35. von Scheidt W, Zugck C, Pauschinger M, Hambrecht R, Bruder $\mathrm{O}$, Hartmann A, et al. Characteristics, management modalities and outcome in chronic systolic heart failure patients treated in tertiary care centers: Results from the EVIdence based TreAtment in Heart Failure (EVITA-HF) registry. Clin Res Cardiol 2014; 103: $1006-1014$.

\section{Supplementary Files}

Please find supplementary file(s);

http://dx.doi.org/10.1253/circrep.CR-18-0004 